\title{
Turing degree spectra of minimal subshifts
}

\author{
Mike Hochman and Pascal Vanier*
}

July 10, 2018

\begin{abstract}
Subshifts are shift invariant closed subsets of $\Sigma^{\mathbb{Z}^{d}}$, minimal subshifts are subshifts in which all points contain the same patterns. It has been proved by Jeandel and Vanier that the Turing degree spectra of nonperiodic minimal subshifts always contain the cone of Turing degrees above any of its degree. It was however not known whether each minimal subshift's spectrum was formed of exactly one cone or not. We construct inductively a minimal subshift whose spectrum consists of an uncountable number of cones with disjoint base.
\end{abstract}

A $\mathbb{Z}^{d}$-subshift is a closed shift invariant subset of $\Sigma^{\mathbb{Z}^{d}}$. Subshifts may be seen as sets of colorings of $\mathbb{Z}^{d}$, with a finite number of colors, avoiding some set of forbidden patterns. Minimal subshifts are subshifts containing no proper subshift, or equivalently subshifts in which all configurations have the same patterns. They are fundamental in the sense that all subshifts contain at least a minimal subshift.

Degrees of unsolvability of subshifts have now been studied for a few years, Cenzer, Dashti, and King [CDK08]; Cenzer, Dashti, Toska, and Wyman [CDTW10; CDTW12] studied computability of one dimensional subshifts and proved some results about their Turing degree spectra: the Turing degree spectrum of a subshift is the set of Turing degrees of its points, see Kent and Lewis [KL10]. Simpson [Sim11], building on the work of Hanf [Han74]; Myers [Mye74], noticed that the Medvedev and Muchnik degrees of subshifts of finite type (SFTs) are the same as the Medvedev degrees of $\Pi_{1}^{0}$ classes: $\Pi_{1}^{0}$ classes are the subsets of $\{0,1\}^{\mathbb{N}}$ for which there exists a Turing machine halting only on oracles not in the subset.

Subsequently Jeandel and Vanier [JV13] focused on Turing degree spectra of different classes of multidimensional subshifts: SFTs, sofic and effective subshifts. They proved in particular that the Turing degree spectra of SFTs are almost the same as the spectra of $\Pi_{1}^{0}$ classes: adding a computable point to the spectrum of any $\Pi_{1}^{0}$ class, one can construct an SFT with this spectrum. In order to prove that one cannot get a stronger statement, they proved that the

\footnotetext{
${ }^{*}$ This work was partially supported by grants EQINOCS ANR 11 BS02 004 03, TARMAC ANR 12 BS02 00701 and ISF grant 1409/11.
} 
spectrum of any non-periodic minimal subshift contains the cone above any of its degrees:

Theorem 1 (Jeandel and Vanier [JV13]). Let $X$ be a minimal non-finite subshift (i.e. non-periodic in at least one direction). For any point $x \in X$ and any degree $\mathbf{d} \geq_{T} \operatorname{deg}_{T} x$, there exists a point $y \in X$ such that $\mathbf{d}=\operatorname{deg}_{T} y$.

Minimal subshifts are in particular interesting since any subshift contains a minimal subshift [Bir12]. Here we answer the followup question of whether a minimal subshift always corresponds to a single cone or if there exists one with at least two cones of disjoint base. It is quite easy to prove the following theorem:

Theorem 2. For any Turing degree $\operatorname{deg}_{T} d$, there exists a minimal subshift $X$ such that the set of Turing degrees of the points of $X$ is a cone of base $\operatorname{deg}_{T} d$.

For instance the spectrum of a Sturmian subshift [MH40] with an irrational angle is the cone whose base is the degree of the angle of the rotation. The theorem can also be seen as a corollary of Miller's proof [Mil12][Proposition 3.1] of a result on Medvedev degrees.

In this paper, we prove the following result:

Theorem 3. There exist a minimal subshift $X \subset\{0,1\}^{\mathbb{Z}}$ and points $x_{z} \in X$ with $z \in\{0,1\}^{\mathbb{N}}$ such that for any $z \neq z^{\prime} \in\{0,1\}^{\mathbb{N}}, \operatorname{deg}_{T} x_{z}$ and $\operatorname{deg}_{T} x_{z^{\prime}}$ are incomparable and such that there exists no point $y \in X$ with $\operatorname{deg}_{T} y \leq_{T} \operatorname{deg}_{T} x_{z}$ and $\operatorname{deg}_{T} y \leq_{T} \operatorname{deg}_{T} x_{z^{\prime}}$.

The subshift constructed in this proof is not effective and cannot be "effectivized", since minimal effective subshifts always contain a computable point and thus their spectra are the whole set of Turing degrees when they are non-periodic.

\section{Preliminary definitions}

We give here some standard definitions and facts about subshifts, one may consult the book of Lind and Marcus [LM95] for more details.

Let $\Sigma$ be a finite alphabet, its elements are called symbols, the $d$-dimensional full shift on $\Sigma$ is the set $\Sigma^{\mathbb{Z}^{d}}$ of all maps (colorings) from $\mathbb{Z}^{d}$ to the $\Sigma$ (the colors). For $v \in \mathbb{Z}^{d}$, the shift functions $\sigma_{v}: \Sigma^{\mathbb{Z}^{d}} \rightarrow \Sigma^{\mathbb{Z}^{d}}$, are defined locally by $\sigma_{v}\left(c_{x}\right)=$ $c_{x+v}$. The full shift equipped with the distance $d(x, y)=2^{-\min \left\{\|v\| \mid v \in \mathbb{Z}^{d}, x_{v} \neq y_{v}\right\}}$ is a compact metric space on which the shift functions act as homeomorphisms. An element of $\Sigma^{\mathbb{Z}^{d}}$ is called a configuration.

Every closed shift-invariant (invariant by application of any $\sigma_{v}$ ) subset $X$ of $\Sigma^{\mathbb{Z}^{d}}$ is called a subshift. An element of a subshift is called a point of this subshift.

Alternatively, subshifts can be defined with the help of forbidden patterns. A pattern is a function $p: P \rightarrow \Sigma$, where $P$, the support, is a finite subset of $\mathbb{Z}^{d}$. We say that a configuration $x$ contains a pattern $p: P \rightarrow$ Sigma, or 
equivalently that the pattern $p$ appears in $x$, if there exists $z \in \mathbb{Z}^{d}$ such that $x_{\mid z+P}=p$.

Let $\mathcal{F}$ be a collection of forbidden patterns, the subset $X_{F}$ of $\Sigma^{\mathbb{Z}^{d}}$ containing the configurations having nowhere a pattern of $F$. More formally, $X_{\mathcal{F}}$ is defined by

$$
X_{\mathcal{F}}=\left\{x \in \Sigma^{\mathbb{Z}^{d}} \mid \forall z \in \mathbb{Z}^{d}, \forall p \in F, x_{\mid z+P} \neq p\right\} .
$$

In particular, a subshift is said to be a subshift of finite type (SFT) when it can be defined by a collection of forbidden patterns that is finite. Similarly, an effective subshift is a subshift which can be defined by a recursively enumerable collection of forbidden patterns. A subshift is sofic if it is the image of an SFT by a letter by letter function.

Definition 1 (Minimal subshift). A subshift $X$ is called minimal if it verifies one of the following equivalent conditions:

- There is no subshift $Y$ such that $Y \subsetneq X$.

- All the points of $X$ contain the same patterns.

- It is the closure of the orbit of any of its points.

We will use the two latter conditions.

For $x, y \in\{0,1\}^{\mathbb{N}}$, we say that $x \leq_{T} y$ if there exists a Turing machine $M$ such that $M$ with oracle $y$ computes $x$. Of course $x \equiv_{T} y$ when we have both $x \leq_{T} y$ and $y \leq_{T} x$. The Turing degree of $x$ is the equivalence class of $x$ with respect to $\equiv_{T}$. More details can be found in Rogers [Rog67]. We call recursive operator a partial function $\phi:\{0,1\}^{\mathbb{N}} \rightarrow\{0,1\}^{\mathbb{N}}$ corresponding to a Turing machine whose input is its oracle and output is an infinite sequence. We say that the function is undefined on the inputs on which the Turing machine does not output an infinite sequence of bits.

For a possibly infinite word $w=w_{0} \ldots w_{n}$, we denote $w_{[i, j]}=w_{i} \ldots w_{j}$.

\section{Minimal subshifts with several cones}

Lemma 2.1. There exists a countable set $\mathcal{C} \subseteq\{0,1\}^{\mathbb{N}}$ such that for any two recursive partial operators $\phi_{1}, \phi_{2}:\{0,1\}^{\mathbb{N}} \rightarrow\{0,1\}^{\mathbb{N}}$ and two distinct words $L=\left\{w_{1}, w_{2}\right\} \subseteq\{0,1\}^{*}$. There exist two words $w_{1}^{\prime}, w_{2}^{\prime} \in L^{*}$ starting respectively with $w_{1}$ and $w_{2}$ such that we have one of the following:

(a) either for any pair $x, y \in\{0,1\}^{\mathbb{N}}, \phi_{1}\left(w_{1}^{\prime} x\right)$ differs from $\phi_{2}\left(w_{2}^{\prime} y\right)$ when they are both defined,

(b) or for any pair $x, y \in\{0,1\}^{\mathbb{N}}, \phi_{1}\left(w_{1}^{\prime} x\right)=\phi_{2}\left(w_{2}^{\prime} y\right) \in \mathcal{C}$ when both defined.

Proof. Let $M_{1}, M_{2}$ be the Turing machines computing the functions $x \mapsto \phi_{1}\left(w_{1} x\right), x \mapsto$ $\phi_{2}\left(w_{2} x\right)$ respectively. When restricting ourselves to inputs on which both operators are defined, it is quite clear that: 
- either there exists some sequences $x, y \in L^{*}$ such that $M_{1}(x)$ 's output differs from $M_{2}(y)$ 's output at some step,

- or the outputs of both machines $M_{1}, M_{2}$ do not depend on their inputs on their respective domains and are equal, in this latter case, we are in case b. We define $\mathcal{C}$ to be the set of these outputs.

In the former case, there exist prefixes $w_{1}^{\prime}$ and $w_{2}^{\prime}$ of $w_{1} x$ and $w_{2} y$ such that the partial outputs of $M_{1}$ once it has read $w_{1}^{\prime}$ already differs from the partial output of $M_{2}$ once it has read $w_{2}^{\prime}$.

In the latter case, one may take $w_{1}^{\prime}=w_{1}$ and $w_{2}^{\prime}=w_{2} . \mathcal{C}$ is countable since there is a countable number of quadruples $\phi_{1}, \phi_{2}, w_{1}, w_{2}$.

Theorem 4. There exists a minimal subshift $X \subseteq\{0,1\}^{\mathbb{N}}$ whose set of Turing degrees contains $2^{\aleph_{0}}$ disjoint cones of Turing degrees.

Note that the following proof is in no way effective. As a matter of fact, all effective minimal subshifts contain some recursive point [BJ10], and their set of Turing degrees is the cone of all degrees.

Proof. We construct a sequence of sofic subshifts $\left(X_{i}\right)_{i \in \mathbb{N}}$ such that $X_{i+1} \subseteq X_{i}$ and such that the limit $X=\bigcap_{i \in \mathbb{N}} X_{i}$ is minimal. In the process of constructing the $X_{i}$, which will be formed of concatenations of allowed words $x_{1}^{i}, \ldots, x_{k}^{i}$, we ensure that no extensions of two distinct words may compute an identical sequence with any of the first $i$ Turing machines. At the same time, we make sure that all allowed words of level $i+1$ contain all words of level $i$, thus enforcing the minimality of the limit $X$. We also have to avoid that the limit $X$ contains a computable point.

Let $\left(\mathcal{M}_{i}\right)_{i \in \mathbb{N}}$ be an enumeration of all minimal subshifts containing a point of the set $\mathcal{C}$ defined in lemma 2.1. Such an enumeration exists since $\mathcal{C}$ is countable and minimal subshifts are the closure of any of their points. We will also need an enumeration $\left(\phi_{i}\right)_{i \in \mathbb{N}}$ of the partial recursive operators from $\{0,1\}^{\mathbb{N}}$ to $\{0,1\}^{\mathbb{N}}$.

Now let us define the sequence of sofic shifts $\left(X_{i}\right)_{i \in \mathbb{N}}$. Each of these subshifts will be the shift invariant closure of the biinfinite words formed by concatenations of words of some language $L_{i}$ which here will be finite languages. We define $X_{0}=\{0,1\}^{\mathbb{N}}$ that is to say $X_{0}$ is generated by $L_{0}=\left\{w_{0}=0, w_{1}=1\right\}$. Let us now give the induction step. At each step, $L_{i+1}$ will contain $2^{i+1}$ words $w_{0 \ldots 0}, \ldots, w_{1 \ldots 1}$, the indices being binary words of length $i+1$, which will verify the following conditions:

1. The words $w_{b 0}, w_{b 1}$ of $L_{i+1}$ start with the word $w_{b}$ of $L_{i}$.

2. The words $w_{b}$ with $b \in\{0,1\}^{i+1}$ of $L_{i+1}$ each contain all the words of $w_{b^{\prime}}$ with $b^{\prime} \in\{0,1\}^{i}$ of $L_{i}$.

3. For any two words $w_{b}, w_{b^{\prime}}$ of $L_{i+1}$ and for all $j, j^{\prime} \leq i$ :

- Either for all $x, y \in L_{i}^{\omega}, \phi_{j}\left(w_{b} x\right) \neq \phi_{j^{\prime}}\left(w_{b^{\prime}} y\right)$ when both defined,

- Or for all $x, y \in L_{i}^{\omega}, \phi_{j}\left(w_{b} x\right), \phi_{j^{\prime}}\left(w_{b^{\prime}} y\right)$ are in $\mathcal{C}$ when defined. 
4. The words $w_{b 0}, w_{b 1}$ do not appear in any configuration of $\mathcal{M}_{j}$, for all $j \leq i$.

Conditions 1 and 2 are easy to ensure: just make $w_{b a}$ start with $w^{\prime}=$ $w_{b} w_{0 \ldots 0} \ldots w_{1 \ldots 1}$. We then use Lemma 2.1 to extend $w^{\prime}$ into a word $w^{\prime \prime}$ verifying condition 3 , this is done several times, once for every quadruple $w, w^{\prime}, j, j^{\prime}$. And finally, since $X_{i}$ is not minimal, we can extend $w^{\prime \prime}$ so that it contains a pattern appearing in none of the $\mathcal{M}_{j}$ 's for $j \leq i$, to obtain condition. Now we can extend $w^{\prime \prime}$ with two different words thus obtaining $w_{b 0}$ and $w_{b 1}$.

Now let's check that this leads to the desired result:

- $X=\bigcap X_{i}$ is a countable intersection of compact shift-invariant spaces, it is compact and shift-invariant, thus a subshift.

- Any pattern $p$ appearing in some point of $X$ is contained in a pattern $w_{b}$, with $b \in\{0,1\}^{i}$ for some $i$, by construction (condition 2), all $w_{b^{\prime}}$ with $b \in\{0,1\}^{i+1}$ contain $w_{b}$. Therefore, all points of $X$, since they are contained in $X_{i+1}$, contain $w_{b}$ and hence $p$. So $X$ is minimal.

- For all $z \in\{0,1\}^{\mathbb{N}}$, define the points $x_{z}=\lim _{i \rightarrow \infty} x_{z[0, i]}$, they are in $X$ because they belong to each $X_{i}$. Condition 3 ensures that if two of them compute the same sequence $y \in\{0,1\}^{\mathbb{N}}$, then this sequence is in $\mathcal{C}$. And condition 2 ensures that no point of $X$ belongs to a minimal subshift containing a point of $\mathcal{C}$.

It is quite straightforward to transform this proof in order to get a subshift on $\{0,1\}^{\mathbb{Z}}$ instead of $\{0,1\}^{\mathbb{N}}$ and obtain the following corollary:

Corollary 5. For any dimension $d$, there exists a minimal subshift $X \subseteq\{0,1\}^{\mathbb{Z}^{d}}$ whose set of Turing degrees contains $2^{\aleph_{0}}$ disjoint cones of Turing degrees.

\section{References}

[Bir12] M.-G. D. Birkhoff. "Quelques théorêmes sur le mouvement des systémes dynamiques". In: Bulletin de la SMF 40 (1912), pp. 305323.

[BJ10] A. Ballier and E. Jeandel. "Computing (or not) quasi-periodicity functions of tilings". In: Second symposium on Cellular Automata (JAC 2010). 2010.

[CDK08] D. Cenzer, A. Dashti, and J. L. F. King. "Computable symbolic dynamics". In: Mathematical Logic Quarterly 54.5 (2008), pp. 460469. DOI: $10.1002 / \mathrm{malq} .200710066$.

[CDTW10] D. Cenzer, A. Dashti, F. Toska, and S. Wyman. "Computability of Countable Subshifts". In: Computability in Europe (CiE). Vol. 6158. Lecture Notes in Computer Science. 2010, pp. 88-97. DOI: $10.1007 / 978-3-642-13962-8 \_10$. 
[CDTW12] D. Cenzer, A. Dashti, F. Toska, and S. Wyman. "Computability of Countable Subshifts in One Dimension". In: Theory of Computing Systems (2012). DOI: 10.1007/s00224-011-9358-z.

[Han74] W. Hanf. "Non Recursive Tilings of the Plane I". In: Journal of Symbolic Logic 39.2 (June 1974), pp. 283-285.

[JV13] E. Jeandel and P. Vanier. "Turing degrees of multidimensional SFTs". In: Theoretical Computer Science 505.0 (2013). Theory and Applications of Models of Computation 2011, pp. 81 -92. ISSN: 0304-3975. DOI: http://dx.doi.org/10.1016/j.tcs.2012.08.027. URL: http://www.sciencedirect.com/science/article/pii/S0304397512008031.

[KL10] T. Kent and A. E. M. Lewis. "On the degree spectrum of a $\Pi_{1}^{0}$ class". In: Transactions of the American Mathematical Society 362 (2010), pp. 5283-5319.

[LM95] D. Lind and B. Marcus. An Introduction to Symbolic Dynamics and Coding. New York, NY, USA: Cambridge University Press, 1995. ISBN: 0-521-55900-6.

[MH40] H. M. Morse and G. A. Hedlund. "Symbolic Dynamics II. Sturmian Trajectories". In: American Journal of Mathematics 62.1 (1940), pp. 1-42.

[Mil12] J. S. Miller. "Two Notes on subshifts". In: Proceedings of the American Mathematical Society 140.5 (2012), pp. 1617-1622. DOI: 10.1090/S0002-9939-2011-11000-1.

[Mye74] D. Myers. "Non Recursive Tilings of the Plane II". In: Journal of Symbolic Logic 39.2 (June 1974), pp. 286-294.

[Rog67] H. Rogers. Theory of recursive functions and effective computability. McGraw-Hill series in higher mathematics. McGraw-Hill, 1967. URL: http://books . google.fr/books?id=nAkzAAAAMAJ.

[Sim11] S. G. Simpson. "Medvedev Degrees of 2-Dimensional Subshifts of Finite Type". In: Ergodic Theory and Dynamical Systems (2011). 